\title{
LoCuSS: A COMPARISON OF SUNYAEV-ZEL'DOVICH EFFECT AND GRAVITATIONAL-LENSING MEASUREMENTS OF GALAXY CLUSTERS
}

\author{
Daniel P. Marrone ${ }^{1,2,15}$, Graham P. Smith ${ }^{3}$, Johan Richard ${ }^{4,5}$, Marshall Joy ${ }^{6}$, Massimiliano Bonamente ${ }^{7}$, \\ Nicole Hasler ${ }^{7}$, Victoria Hamilton-Morris ${ }^{3}$, Jean-Paul KNeib ${ }^{8}$, Thomas Culverhouse ${ }^{1,2}$, John E. Carlstrom ${ }^{1,2,9,10}$, \\ Christopher Greer ${ }^{1,2}$, David Hawkins ${ }^{11}$, Ryan Hennessy ${ }^{1,2}$, James W. Lamb ${ }^{11}$, Erik M. Leitch ${ }^{1,2}$, Michael LoH ${ }^{1,8}$, \\ Amber Miller $^{12,13}$, Tony Mroczkowski ${ }^{12,14}$, Stephen Muchovej ${ }^{11,14}$, Clem Pryke $^{1,2,10}$, Matthew K. Sharp ${ }^{1,9}$, \\ AND DAVID WOODY ${ }^{11}$ \\ ${ }^{1}$ Kavli Institute for Cosmological Physics, Department of Astronomy and Astrophysics, University of Chicago, Chicago, IL 60637, USA \\ ${ }^{2}$ Department of Astronomy and Astrophysics, University of Chicago, Chicago, IL 60637, USA \\ ${ }^{3}$ School of Physics and Astronomy, University of Birmingham, Edgbaston, Birmingham, B15 2TT, UK \\ ${ }^{4}$ California Institute of Technology, Mail Code 105-24, Pasadena, CA 91125, USA \\ ${ }^{5}$ Department of Physics, Durham University, South Road, Durham, DH1 3LE, UK \\ ${ }^{6}$ Space Science Office, VP62, NASA/Marshall Space Flight Center, Huntsville, AL 35812, USA \\ ${ }^{7}$ Department of Physics, University of Alabama, Huntsville, AL 35812, USA \\ ${ }^{8}$ Laboratoire d'Astrophysique de Marseilles, OAMP, CNRS-Université Aix-Marseilles, 38 rue Frédéric Joliot-Curie, 13388 Marseilles Cedex 13, France \\ ${ }^{9}$ Department of Physics, University of Chicago, Chicago, IL 60637, USA \\ ${ }^{10}$ Enrico Fermi Institute, University of Chicago, Chicago, IL 60637, USA \\ ${ }^{11}$ Owens Valley Radio Observatory, California Institute of Technology, Big Pine, CA 93513, USA \\ ${ }^{12}$ Columbia Astrophysics Laboratory, Columbia University, New York, NY 10027, USA \\ ${ }^{13}$ Department of Physics, Columbia University, New York, NY 10027, USA \\ ${ }^{14}$ Department of Astronomy, Columbia University, New York, NY 10027, USA \\ Received 2009 April 7; accepted 2009 July 6; published 2009 August 10
}

\begin{abstract}
We present the first measurement of the relationship between the Sunyaev-Zel'dovich effect (SZE) signal and the mass of galaxy clusters that uses gravitational lensing to measure cluster mass, based on $14 \mathrm{X}$-ray luminous clusters at $z \simeq 0.2$ from the Local Cluster Substructure Survey. We measure the integrated Compton $y$-parameter, $Y$, and total projected mass of the clusters $\left(M_{\mathrm{GL}}\right)$ within a projected clustercentric radius of $350 \mathrm{kpc}$, corresponding to mean overdensities of $4000-8000$ relative to the critical density. We find self-similar scaling between $M_{\mathrm{GL}}$ and $Y$, with a scatter in mass at fixed $Y$ of $32 \%$. This scatter exceeds that predicted from numerical cluster simulations, however, it is smaller than comparable measurements of the scatter in mass at fixed $T_{X}$. We also find no evidence of segregation in $Y$ between disturbed and undisturbed clusters, as had been seen with $T_{X}$ on the same physical scales. We compare our scaling relation to the Bonamente et al. relation based on mass measurements that assume hydrostatic equilibrium, finding no evidence for a hydrostatic mass bias in cluster cores $\left(M_{\mathrm{GL}}=0.98 \pm 0.13 M_{\mathrm{HSE}}\right)$, consistent with both predictions from numerical simulations and lensing/ $\mathrm{X}$-ray-based measurements of mass-observable scaling relations at larger radii. Overall our results suggest that the SZE may be less sensitive than X-ray observations to the details of cluster physics in cluster cores.
\end{abstract}

Key words: cosmology: observations - galaxies: clusters: general - gravitational lensing

\section{INTRODUCTION}

The Sunyaev-Zel'dovich effect (SZE) is a weak distortion of the cosmic microwave background (CMB) spectrum introduced as $\mathrm{CMB}$ photons propagate through foreground galaxy clusters (Sunyaev \& Zel'dovich 1970, 1972). Because this signal does not suffer cosmological dimming and is expected to closely track cluster mass, the SZE is a potentially powerful tool for producing large, mass-limited galaxy cluster samples that can be used to constrain dark energy, under the proviso that the relationship between cluster mass and the observed signal is well calibrated (Carlstrom et al. 2002).

The relationship between the SZE observable, the integrated Compton $y$-parameter, $Y$, and cluster mass is difficult to calibrate because of the difficulty of measuring mass directly. To date all examinations of the mass $-Y$ relationship have assumed hydrostatic equilibrium (HSE) when converting SZE or $\mathrm{X}$-ray measurements of the intracluster gas into estimates of the total mass $\left(M_{\mathrm{HSE}}\right.$; e.g., Morandi et al. 2007; Bonamente

\footnotetext{
15 Jansky Fellow, National Radio Astronomy Observatory.
}

et al. 2008). These methods measure scaling relations between closely related quantities derived from the same data; deviations in one parameter will therefore inevitably be correlated with the other, and the true intrinsic scatter will likely be underestimated. Furthermore, both simulations (e.g., Nagai et al. 2007; Jeltema et al. 2008; Piffaretti \& Valdarnini 2008; Lau et al. 2009) and observations (Mahdavi et al. 2008) suggest that the HSE assumption is incorrect; non-thermal sources of pressure support may bias $M_{\mathrm{HSE}}$ or increase the scatter with $Y$.

The scatter in mass at fixed $Y$ is an important quantity to determine in preparation for the SZE cluster surveys designed to constrain the dark energy equation of state, as scatter in the mass-observable relation is generally degenerate with the cosmological parameters of interest (e.g., Smith et al. 2003; Stanek et al. 2006). These surveys will rely on self-calibration techniques to build cluster mass functions from SZE-selected cluster catalogs, however, these techniques can be rendered ineffective if the scatter is much larger than the few percent predicted by simulations (da Silva et al. 2004; Motl et al. 2005; Nagai 2006). At present, there are only weak observational limits, but independent measurements of the mass $-Y$ 
Table 1

Cluster Sample

\begin{tabular}{|c|c|c|c|c|c|c|c|}
\hline Cluster & Redshift & $\begin{array}{c}Y \\
\left(10^{-10}\right)\end{array}$ & $\begin{array}{c}Y D_{\mathrm{A}}^{2} \\
\left(10^{-5} \mathrm{Mpc}^{2}\right)\end{array}$ & $\begin{array}{l}\text { SZE } \\
\text { Ref. }\end{array}$ & $\begin{array}{c}M_{\mathrm{GL}} \\
\left(10^{14} M_{\odot}\right) \\
\end{array}$ & $\begin{array}{l}\text { Lensing } \\
\text { Ref. }\end{array}$ & Classification \\
\hline A 68 & 0.255 & $0.55 \pm 0.08$ & $3.67 \pm 0.52$ & 1 & $3.48 \pm 0.07$ & 2,3 & Disturbed \\
\hline A 209 & 0.206 & $0.94 \pm 0.14$ & $4.57 \pm 0.67$ & 4 & $1.23 \pm 0.39$ & 2 & Disturbed \\
\hline A 267 & 0.230 & $0.53 \pm 0.06$ & $3.08 \pm 0.34$ & 1 & $2.20 \pm 0.34$ & 2 & Disturbed \\
\hline A 383 & 0.188 & $0.39 \pm 0.04$ & $1.61 \pm 0.16$ & 4 & $3.71 \pm 0.82$ & 2 & Undisturbed \\
\hline A 611 & 0.288 & $0.39 \pm 0.04$ & $3.13 \pm 0.34$ & 1 & $2.12 \pm 0.05$ & 5 & Undisturbed \\
\hline A 773 & 0.217 & $1.03 \pm 0.11$ & $5.40 \pm 0.57$ & 1 & $4.03 \pm 0.12$ & 2,5 & Disturbed \\
\hline Z 2701 & 0.214 & $0.28 \pm 0.03$ & $1.46 \pm 0.16$ & 4 & $1.92 \pm 0.07$ & 5 & Disturbed \\
\hline A 1413 & 0.143 & $1.83 \pm 0.26$ & $4.90 \pm 0.70$ & 1 & $2.59 \pm 0.50$ & 5 & Undisturbed \\
\hline A 1689 & 0.181 & $1.86 \pm 0.15$ & $7.51 \pm 0.60$ & 1 & $7.44 \pm 0.05$ & 6 & Disturbed \\
\hline A 1763 & 0.288 & $0.56 \pm 0.06$ & $3.10 \pm 0.32$ & 4 & $1.42 \pm 0.54$ & 2 & Disturbed \\
\hline A 1835 & 0.253 & $1.03 \pm 0.07$ & $6.82 \pm 0.48$ & 1 & $3.35 \pm 0.06$ & 2,5 & Undisturbed \\
\hline A 2218 & 0.171 & $1.12 \pm 0.10$ & $4.23 \pm 0.38$ & 1 & $4.23 \pm 0.09$ & 2 & Disturbed \\
\hline A 2219 & 0.228 & $1.12 \pm 0.05$ & $6.27 \pm 0.26$ & 4 & $3.48 \pm 0.07$ & 2 & Disturbed \\
\hline A 2537 & 0.297 & $0.42 \pm 0.03$ & $3.47 \pm 0.24$ & 4 & $1.75 \pm 0.90$ & 7 & Disturbed \\
\hline
\end{tabular}

Notes. $Y$ and $M_{\mathrm{GL}}$ measured at $350 \mathrm{kpc}$ radius. Morphological classification from lensing and X-ray data. See S05 and Smith \& Taylor (2008).

References. (1) Bonamente et al. 2008; (2) S05; (3) Richard et al. 2007; (4) this work; (5) J. Richard et al. 2009, in preparation; (6) Limousin et al. 2007; (7) V. Hamilton-Morris et al. 2009, in preparation.

scatter can be used to refine the self-calibration (Lima \& Hu 2005).

The Local Cluster Substructure Survey (LoCuSS ${ }^{16}$; G. P. Smith et al. 2009, in preparation) is a morphologically unbiased survey of X-ray luminous galaxy clusters in a narrow redshift range $(0.15<z<0.3)$. The survey unites a variety of probes of total mass, intracluster gas, and cluster member galaxies (e.g., Smith et al. 2005, hereafter S05; Zhang et al. 2008; Okabe et al. 2009). One of the main goals of the survey is to measure the shape, normalization, and scatter of cluster massobservable relations as an input to cosmological cluster studies. Here, we use a pilot sample of clusters, assembled from S05, Bonamente et al. (2006), and early LoCuSS observations with the Sunyaev-Zel'dovich Array (SZA), to compare the SZE signal with cluster masses derived from gravitational lensing $\left(M_{\mathrm{GL}}\right)$ and thus to construct the first mass $-Y$ relation that is independent of the assumption of HSE. We summarize the observations and modeling in Section 2. The $M_{\mathrm{GL}}-Y$ relationship, scatter, and its implications are discussed in Section 3. We assume $\Omega_{M}=0.3$, $\Omega_{\Lambda}=0.7$, and $h=0.7$; the median cluster redshift is $z=0.222$, at which $1^{\prime \prime}$ corresponds to a physical scale of $3.6 \mathrm{kpc}$.

\section{OBSERVATIONS AND MODELING}

We analyze SZE and gravitational-lensing observations of 14 clusters, listed in Table 1. SZE results for eight of these come from LaRoque et al. (2006) and Bonamente et al. (2006, 2008), the observational details being found in LaRoque et al. New SZE measurements of A 209, A 383, A 1763, A 2219, A 2537, and Z 2701 were obtained at $31 \mathrm{GHz}$ using the SZA, which has baselines of 350-1300 $\lambda$ for SZE sensitivity (angular scales of $110^{\prime \prime}-7^{\prime}$ ) and 2-7.5 $\mathrm{k} \lambda$ for radio source removal. A detailed description of SZA observations and analysis methods can be found in Muchovej et al. (2007). Typical integration times were $\sim 30-40 \mathrm{hr}$ per cluster, and the rms noise per beam $\sim 0.2 \mathrm{mJy}$. In six of 14 clusters, we detect radio sources within an arcminute of the cluster center, these sources were presented in Coble et al. (2007).

Our interferometric observations do not measure directly the total SZE flux of the cluster within an aperture, this must be

\footnotetext{
16 http://www.sr.bham.ac.uk/locuss
}

derived from a model cluster profile. As in previous works, we have modeled these clusters with an isothermal $\beta$-model, which has been shown to provide $Y$ measurements indistinguishable from those from a simulation-motivated pressure profile for radii smaller than $r_{\text {vir }} / 3$ (Mroczkowski et al. 2009). The shape parameters for the $\beta$-model and an isothermal X-ray temperature were jointly derived from the SZE data and from Chandra $\mathrm{X}$-ray images after applying a $100 \mathrm{kpc}$ core cut (LaRoque et al. 2006). Best-fit cluster parameters were obtained using the Markov chain Monte Carlo method described in Bonamente et al. (2004). The fluxes of detected radio sources and central sources present in the $1.4 \mathrm{GHz}$ NVSS catalog (Condon et al. 1998) but undetected at $31 \mathrm{GHz}$ are included as parameters in the Markov chains and marginalized over.

Details of the gravitational-lensing mass measurements are described by S05 and Richard et al. (2007); the source of each measurement is listed in Table 1. Briefly, the mass measurements are based on Hubble Space Telescope (HST) imaging of the cluster cores, ground-based spectroscopy of multiply imaged galaxies identified in these data, and by weakly sheared background galaxies. These data constrain parameterized models of the projected mass distribution in the cluster cores comprising one or more cluster-scale mass components (to describe the dark matter and intracluster gas) plus typically $\sim 30$ galaxy-scale mass components per cluster. Measurements of the projected cluster mass and the uncertainty on the mass are then obtained by integrating the mass distributions of the family of models within the chosen confidence interval in parameter space (in this Letter we quote errors at $68 \%$ confidence) out to the chosen radius.

S05 (see also Smith \& Taylor 2008) used the structure of the cluster mass distributions inferred from these lens models - the substructure fraction, $f_{\text {sub }}$-in conjunction with Chandra observations to classify the cluster cores as "disturbed" or "undisturbed." The most straightforward criterion in this classification was the offset between the peaks of the X-ray emission and the lensing-based mass map, in the sense that a statistically significant offset indicates that the cluster core is disturbed in some way, probably due to a cluster-cluster merger (see also Sanderson et al. 2009). We adopt the classification of Smith \& Taylor (2008) in this Letter and apply it to the five additional clusters in our sample (Table 1). 


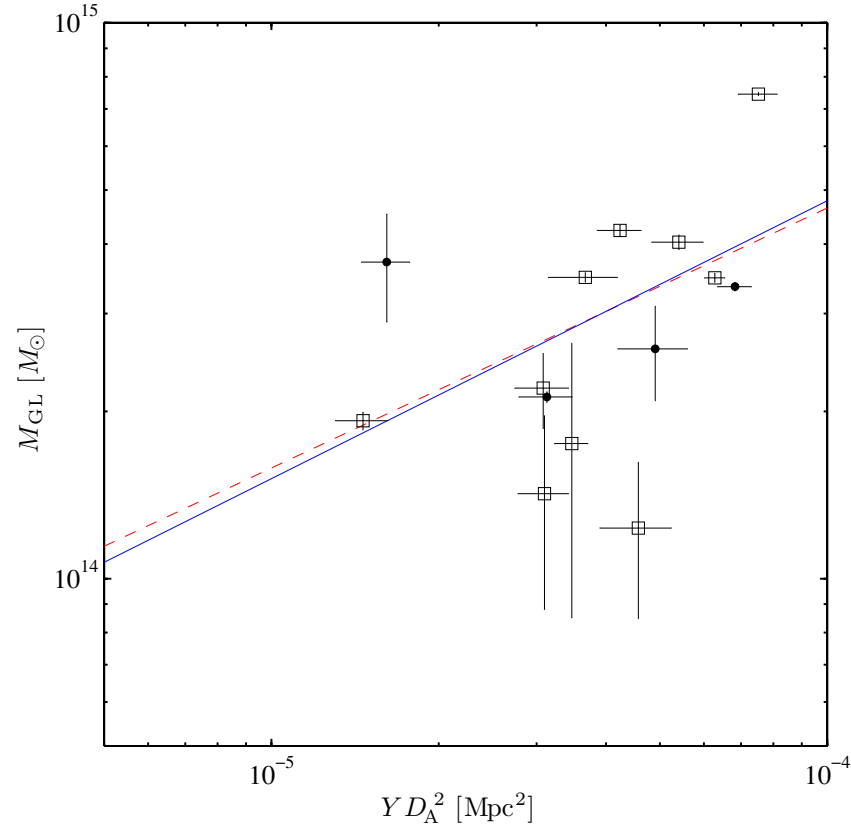

Figure 1. Scaling between projected mass $\left(M_{\mathrm{GL}}\right)$ based on gravitational lens models and $Y$, both measured within a projected clustercentric radius of $350 \mathrm{kpc}$. Clusters classified as undisturbed in Table 1 are shown as filled circles, disturbed clusters are shown as open boxes. The solid line shows the scaling relation when the slope is fixed to the self-similar value $(\beta=0.5)$, the dashed line is obtained when the slope is also a free parameter.

\section{RESULTS AND DISCUSSION}

\subsection{The Mass-Y Scaling Relation}

We first define the aperture within which $M_{\mathrm{GL}}$ and $Y$ are measured. After some experimentation, we chose to retain the aperture used by $\mathrm{S} 05$, a clustercentric radius of $250 \mathrm{~h}^{-1} \mathrm{kpc}$, or $350 \mathrm{kpc}$ in our adopted cosmology. This fixed physical aperture is well matched to the HST/Wide Field Planetary Camera 2 (WFPC2) and Advanced Camera for Surveys fields of view, and is somewhat larger than the typical resolution of our SZE data. The shape of our $\beta$-model fit is jointly determined from both SZA and high-resolution X-ray data and thus is well resolved. $M_{\mathrm{GL}}$ and $Y$ values measured at this radius are listed in Table 1 and plotted in Figure 1.

We fit our projected mass $\left(M_{\mathrm{GL}}\right)$ and $Y$ data points in the $\log \left(Y D_{\mathrm{A}}^{2}\right)-\log \left(M_{\mathrm{GL}}\right)$ plane, where a power-law scaling relation takes the form $\log \left(M_{\mathrm{GL}}\right)=\alpha+\beta \log \left(Y D_{\mathrm{A}}^{2}\right)$. Starting with the self-similar scaling (Kaiser 1986), it can be shown that the relation between mass and $Y$ within a fixed physical radius has a slope $\beta=0.5$ with no dependence on the redshift evolution of the Hubble parameter. We seek to measure the slope and normalization of the scaling relation, as well as the intrinsic scatter between these parameters, and therefore follow the prescription of Weiner et al. (2006) for linear regression in the presence of intrinsic scatter. This Bayesian method includes the intrinsic scatter in the $y$-coordinate $\left(\sigma_{y}\right)$ as a parameter of the fit and maximizes the probability of the model $\left(\alpha, \beta, \sigma_{y}\right)$ given the data and errors. The likelihood takes the form (Equation (A3) of Weiner et al. 2006)

$$
\mathcal{L}=-\Sigma \frac{\left[y_{i}-\left(\alpha+\beta x_{i}\right)\right]^{2}}{\beta^{2} e_{x i}^{2}+e_{y i}^{2}+\sigma_{y}^{2}},
$$

where the $e_{i}$ are the $x$ or $y$ errors in the individual data points.
We perform the regression in two different ways: first, with both the normalization $(\alpha)$ and slope $(\beta)$ of the fit free, we obtain $\alpha=16.5_{-0.9}^{+1.1}$ and $\beta=0.47_{-0.20}^{+0.24}$, with an intrinsic scatter in mass at fixed $Y$ of $\sigma_{M \mid Y}=32 \% \pm 4 \%$. With the slope fixed to the self-similar value $(\beta=0.5)$, we obtain $\alpha=16.68 \pm 0.04$, again with $\sigma_{M \mid Y}=32 \% \pm 4 \%$. Despite large uncertainties, the $M_{\mathrm{GL}}-Y$ slope is consistent with self-similarity. For comparison, Bonamente et al. (2008) and Morandi et al. (2007) found that the $M_{\mathrm{HSE}}-Y$ scaling relation is consistent with selfsimilarity at $r_{2500}$ (the radius where the average interior density is $\Delta=2500$ times the critical density of the universe, typically $30 \%-100 \%$ larger than the radius used here). In contrast, S05 were unable to constrain the slope of the $M_{\mathrm{GL}}-T_{X}$ relation, suggesting that the intrinsic scatter in $M_{\mathrm{GL}}-Y$ is smaller than in $M_{\mathrm{GL}}-T_{X}$.

\subsection{Intrinsic Scatter}

We now compare the intrinsic scatter in the $M_{\mathrm{GL}}-Y$ relation with that in $M_{\mathrm{GL}}-T_{X}$ from S05. We first use our regression method to re-fit their data, taking the opportunity to adopt the theoretical slope $\left(T_{X}=B M_{\mathrm{GL}}^{1 / \beta}, \beta=1\right.$, in their nomenclature) appropriate for this scaling relation within a fixed aperture. With their 10 clusters, we find a scatter in mass of $\sigma_{M \mid T}=41 \% \pm 5 \%$, which is $28 \%$ larger (1.5 $\sigma$ significance) than $\sigma_{M \mid Y}$. Indeed, both $\sigma_{M \mid Y}$ and $\sigma_{M \mid T}$ are large compared to the scatter predicted from numerical simulations (e.g., Nagai 2006), likely due to a combination of modeling uncertainties, astrophysical processes in cluster cores, and projection effects. The effects of modeling uncertainties are hard to quantify but should not be ignored, as both $M_{\mathrm{GL}}$ and $Y$ are derived from parameterized models. To the extent that these models inadequately represent the cluster or underestimate the uncertainty in the derived parameters, our inferred scatter will be artificially increased. Thus, our measured $\sigma_{M \mid Y}$ is an upper limit to the true scatter between mass and $Y$ in cluster cores.

In general, cluster mass measurements based on gravitationallensing and SZE observations are more susceptible to projection effects than those based on X-ray data because the densitysquared dependence of the X-ray emissivity limits contributions from material along the line of sight. Nevertheless, simulations (Holder et al. 2007; Shaw et al. 2008) suggest that the contribution to the SZE signal from projection is unimportant for clusters with $M_{\text {vir }}>10^{14} h^{-1} M_{\odot}$, with the projection effects diminishing further at the small radii used here. Gravitational lensing is sensitive to all mass along the line of sight through the cluster, which will likely increase the scatter in our scaling relation, however, the contribution of correlated large-scale structure to lensing-based mass measurements should decrease at smaller radii in a manner similar to the impact on the SZE signal. We also emphasize that our sample is X-ray selected with no consideration paid to the presence/absence of stronglensing arcs, so it should not suffer the halo orientation bias that boosts the core mass in strong-lensing-selected samples (e.g., Hennawi et al. 2007). Indeed, 5/14 clusters contain no obvious strong-lensing signal. Projection-induced scatter therefore appears insignificant for $Y$ and may be somewhat more important for $M_{\mathrm{GL}}$, although this affects both $\sigma_{M \mid Y}$ and $\sigma_{M \mid T}$.

Given that the mass measurement methods and 9/14 of the clusters are identical between this Letter and S05, we expect the difference between the scatter in these two relations to be dominated by the relative sensitivity of $Y$ and $T_{X}$ to the gas physics of cluster cores. The intracluster medium (ICM) 
in cluster cores is often disturbed by active galactic nucleus activity (e.g., Fabian et al. 2006), and cluster-cluster mergers (Markevitch \& Vikhlinin 2007). For this reason, cluster cores are often excluded when deriving X-ray observables for use as mass proxies (e.g., Arnaud et al. 2005; Kravtsov et al. 2006; Maughan 2007). We therefore ascribe the difference between $\sigma_{M \mid Y}$ and $\sigma_{M \mid T}$ to the difference in the SZE and $X$-ray emissivity dependencies on ICM density and temperature. The SZE is unaffected by isobaric disturbances (excluding relativistic components such as cosmic rays), while the energy-integrated X-ray emissivity varies approximately as the square of the density, which may be significantly perturbed in the core. Pfrommer et al. (2007) found potentially important changes in the SZE and X-ray signals from cool cores in simulations incorporating cosmic rays, but the effect and therefore the induced scatter was found to be larger for X-ray emission. In larger measurement apertures, such as the frequently used $r_{2500}$ and $r_{500}(350 \mathrm{kpc}$ corresponds to $\Delta=4000-8000$ for our sample), core-removed X-ray mass proxies are found to correlate more tightly with mass (e.g., Vikhlinin et al. 2009). We expect that $\sigma_{M \mid Y}$ will also decrease when measured on these scales.

\subsection{Structural Segregation}

S05 found that the normalization of the $M_{\mathrm{GL}}-T_{X}$ relation for disturbed clusters was $\sim 40 \%$ hotter than for undisturbed clusters at $\sim 2.5 \sigma$ (after excising the central region of the $\mathrm{X}$-ray data). We re-fit the $M_{\mathrm{GL}}-Y$ relation to the undisturbed and disturbed subsamples (Table 1) finding no significant difference in normalization between undisturbed and disturbed clusters $\left(\alpha_{\text {undisturbed }}-\alpha_{\text {disturbed }}=0.0 \pm 0.1, \beta\right.$ fixed to 0.5 ). Again, this suggests that the integrated SZE signal, even in cluster cores, is less sensitive to the ICM physics than the X-ray temperature, although the significance of this result is low given the small samples used here and in S05.

\subsection{Hydrostatic Equilibrium}

As discussed in Sections 1 and 3.2, the ICM may be far from HSE; we search for this effect in our data by combining our results with those of Bonamente et al. (2008) who studied the $M_{\mathrm{HSE}}-Y$ relation. Our lensing measurements are sensitive to the projected mass $\left(M_{\text {cyl }}\right)$; we therefore convert them to the spherical masses $\left(M_{\mathrm{sph}}\right)$ required for comparison with the hydrostatic analysis. We do this by assuming that the halo mass distributions are described by the Navarro-Frenk-White profile (NFW) profile (Navarro et al. 1996), which is specified by mass and concentration, $M_{\mathrm{vir}}$ and $c_{\mathrm{vir}}$; given $M_{\mathrm{GL}}$ and $c_{\mathrm{vir}}$ we can than derive an approximate conversion factor $M_{\mathrm{cyl}} / M_{\mathrm{sph}}$ at $350 \mathrm{kpc}$. This conversion factor depends sensitively on $c_{\mathrm{vir}}$, but there is considerable theoretical uncertainty about the mean value and scatter in concentration at fixed mass, and its variation with redshift and cluster mass. Without direct measurements of $c_{\text {vir }}$ for most of our clusters, we marginalize over the range of values observed by Okabe et al. (2009) for 19 clusters from the LoCuSS sample, which have very similar masses and redshifts to our sample. We randomly choose a concentration from the 19 in Okabe et al. (2009) for each of our clusters, calculate the corresponding value of $M_{\mathrm{GL}, \mathrm{sph}}=M_{\mathrm{GL}} \times M_{\mathrm{sph}} / M_{\mathrm{cyl}}$, and fit a scaling relation between the estimated $M_{\mathrm{GL} \text {,sph }}$ and $Y$. We repeat this process thousands of times, and derive a mean normalization of the $M_{\mathrm{GL}, \mathrm{sph}}-Y$ relation of $\alpha_{\mathrm{GL}, \mathrm{sph}}=16.42 \pm$ 0.05 for $\beta=0.5$. This value can be directly compared to the normalization of the $M_{\mathrm{HSE}}-Y$ relation from Bonamente et al.
(2008): $\alpha_{\mathrm{HSE}}=16.425 \pm 0.016$. The statistically insignificant difference between these values implies a mass ratio of $M_{\mathrm{GL}, \mathrm{sph}} /$ $M_{\mathrm{HSE}}=0.98 \pm 0.13$.

Using hydrostatic masses from X-ray observations and weaklensing masses from the literature, Zhang et al. (2008) and Vikhlinin et al. (2009) estimate the weak lensing to hydrostatic mass ratio to be $1.09 \pm 0.08$ and $1.01 \pm 0.11$, respectively, measured at $r_{500}$. Mahdavi et al. (2008), however, demonstrate a radial trend in this ratio between $r_{2500}$ and $r_{500}$, suggestive of a $20 \%$ deficit in hydrostatic masses at $r_{500}$ that cannot be accounted for by the projection of unrelated structures along the line of sight. Our result, interior to the innermost radius examined by Mahdavi et al. (2008), is consistent with their non-detection of a bias in the hydrostatic mass at small radii. The precision of our measurement is limited by the intrinsic scatter in our relation, with a smaller contribution coming from the unknown halo concentration values required to convert between $M_{\mathrm{GL}}$ and $M_{\mathrm{GL} \text {,sph }}$. Future combinations of weak- and strong-lensing measurements, specifically joint strong+weak lens modeling of the clusters combining the Subaru and HST data (G. P. Smith et al. 2009, in preparation) should allow us to evaluate the bias as a function of radius.

\section{SUMMARY}

We have presented the first calibration of the mass $-Y$ relation based on gravitational-lensing measurements of cluster mass, based largely on previously published SZE data and gravitational lens models. We construct the relation at a fixed physical radius of $350 \mathrm{kpc}$ to minimize uncertainties arising from extrapolation of cluster models based on both data sets. In contrast to the mass- $T_{X}$ relation within the same aperture (S05), we succeed in fitting a model with both free slope and normalization. The best-fit slope is consistent with the self-similar prediction, and the intrinsic scatter in mass at fixed $Y$ is $32 \%$, in contrast to the $41 \%$ scatter in mass at fixed $T_{X}$. We also fit the relation to subsamples of disturbed and undisturbed cluster cores but find that the best-fit normalizations for these subsamples are consistent within the errors. Finally, we combine our results with those of Bonamente et al. (2008) to test whether the cluster cores are in HSE. Cluster core masses estimated from lensing and SZE data (assuming HSE) are, on average, consistent within the errors, suggesting that departures from equilibrium are modest in cluster cores. We conclude that the difference between the mass $-Y$ and mass $-T_{X}$ relations is mainly attributable to the relative insensitivity of the $\mathrm{SZ}$ effect to the physics of the ICM in cluster cores. Future articles will explore the mass$Y$ relation at larger radii through weak lensing and in larger samples.

We thank our colleagues in the LoCuSS collaborations for much support, encouragement and help. G.P.S. acknowledges support from the Royal Society and STFC, and thanks the Kavli Institute of Cosmological Physics at the University of Chicago for their hospitality whilst working on this Letter. G.P.S. thanks Alain Blanchard for helpful comments. We gratefully acknowledge the James S. McDonnell Foundation, the National Science Foundation, and the University of Chicago for funding to construct the SZA. The operation of the SZA is supported by NSF Division of Astronomical Sciences through grant AST-0604982. Partial support is provided by NSF Physics Frontier Center grant PHY-0114422 to the Kavli Institute of Cosmological Physics at the University of Chicago, and by 
NSF grants AST-0507545 and AST-05-07161 to Columbia University.

Facilities: SZA, HST (WFPC2)

\section{REFERENCES}

Arnaud, M., Pointecouteau, E., \& Pratt, G. W. 2005, A\&A, 441, 893

Bonamente, M., Joy, M. K., Carlstrom, J. E., Reese, E. D., \& LaRoque, S. J. 2004, ApJ, 614, 56

Bonamente, M., Joy, M., LaRoque, S. J., Carlstrom, J. E., Nagai, D., \& Marrone, D. P. 2008, ApJ, 675, 106

Bonamente, M., Joy, M. K., LaRoque, S. J., Carlstrom, J. E., Reese, E. D., \& Dawson, K. S. 2006, ApJ, 647, 25

Carlstrom, J. E., Holder, G. P., \& Reese, E. D. 2002, ARA\&A, 40, 643

Coble, K., et al. 2007, AJ, 134, 897

Condon, J. J., Cotton, W. D., Greisen, E. W., Yin, Q. F., Perley, R. A., Taylor, G. B., \& Broderick, J. J. 1998, AJ, 115, 1693

da Silva, A. C., Kay, S. T., Liddle, A. R., \& Thomas, P. A. 2004, MNRAS, 348 1401

Fabian, A. C., Sanders, J. S., Taylor, G. B., Allen, S. W., Crawford, C. S., Johnstone, R. M., \& Iwasawa, K. 2006, MNRAS, 366, 417

Hennawi, J. F., Dalal, N., Bode, P., \& Ostriker, J. P. 2007, ApJ, 654, 714

Holder, G. P., McCarthy, I. G., \& Babul, A. 2007, MNRAS, 382, 1697

Jeltema, T. E., Hallman, E. J., Burns, J. O., \& Motl, P. M. 2008, ApJ, 681, 167

Kaiser, N. 1986, MNRAS, 222, 323

Kravtsov, A. V., Vikhlinin, A., \& Nagai, D. 2006, ApJ, 650, 128

LaRoque, S. J., Bonamente, M., Carlstrom, J. E., Joy, M. K., Nagai, D., Reese, E. D., \& Dawson, K. S. 2006, ApJ, 652, 917

Lau, E. T., Kravtsov, A. V., \& Nagai, D. 2009, ApJ, submitted

Lima, M., \& Hu, W. 2005, Phys. Rev. D, 72, 043006

Limousin, M., et al. 2007, ApJ, 668, 643

Mahdavi, A., Hoekstra, H., Babul, A., \& Henry, J. P. 2008, MNRAS, 384, 1567
Markevitch, M., \& Vikhlinin, A. 2007, Phys. Rep., 443, 1

Maughan, B. J. 2007, ApJ, 668, 772

Morandi, A., Ettori, S., \& Moscardini, L. 2007, MNRAS, 379, 518

Motl, P. M., Hallman, E. J., Burns, J. O., \& Norman, M. L. 2005, ApJ, 623, L63

Mroczkowski, T., et al. 2009, ApJ, 694, 1034

Muchovej, S., et al. 2007, ApJ, 663, 708

Nagai, D. 2006, ApJ, 650, 538

Nagai, D., Kravtsov, A. V., \& Vikhlinin, A. 2007, ApJ, 668,

Navarro, J. F., Frenk, C. S., \& White, S. D. M. 1996, ApJ, 462, 563

Okabe, N., Takada, M., Umetsu, K., Futamase, T., \& Smith, G. P. 2009, PASJ, submitted (arXiv:0903.1103)

Pfrommer, C., Enßlin, T. A., Springel, V., Jubelgas, M., \& Dolag, K. 2007, MNRAS, 378, 385

Piffaretti, R., \& Valdarnini, R. 2008, A\&A, 491, 71

Richard, J., et al. 2007, ApJ, 662, 781

Sanderson, A. J. R., Edge, A. C., \& Smith, G. P. 2009, MNRAS, in press (arXiv:0906.1808)

Shaw, L. D., Holder, G. P., \& Bode, P. 2008, ApJ, 686, 206

Smith, G. P., Edge, A. C., Eke, V. R., Nichol, R. C., Smail, I., \& Kneib, J.-P. 2003, ApJ, 590, L79

Smith, G. P., Kneib, J.-P., Smail, I., Mazzotta, P., Ebeling, H., \& Czoske, O. 2005, MNRAS, 359, 417

Smith, G. P., \& Taylor, J. E. 2008, ApJ, 682, L73

Stanek, R., Evrard, A. E., Böhringer, H., Schuecker, P., \& Nord, B. 2006, ApJ, 648, 956

Sunyaev, R. A., \& Zel'dovich, Y. B. 1970, Comments Astrophys. Space Phys., 2,66

Sunyaev, R. A., \& Zel'dovich, Y. B. 1972, Comments Astrophys. Space Phys., 4, 173

Vikhlinin, A., et al. 2009, ApJ, 692, 1033

Weiner, B. J., et al. 2006, ApJ, 653, 1049

Zhang, Y.-Y., Finoguenov, A., Böhringer, H., Kneib, J.-P., Smith, G. P., Kneissl, R., Okabe, N., \& Dahle, H. 2008, A\&A, 482, 451 